\title{
Chipless RFID Sensor Tag for Metal Crack Detection and Characterization
}

\author{
Adi Mahmud Jaya Marindra ${ }^{\circledR}$, Student Member, IEEE, and Gui Yun Tian, Senior Member, IEEE
}

\begin{abstract}
Chipped radio-frequency identification (RFID) sensor systems have been studied for structural health monitoring (SHM) applications. However, the use of chip in sensor tags and its standardized narrowband operation contribute shortcomings in cost, durability, and detection capability. This paper presents a novel use of the frequency signature-based chipless RFID for metal crack detection and characterization operating in ultra-wideband frequency. The vision is to implement a lowcost and high-temperature-resistant passive wireless sensor able to monitor the crack on a metallic structure with multiparameter detection. We propose a chipless RFID sensor tag integrating four tip-loaded dipole resonators as a 4-bit ID encoder and a circular microstrip patch antenna (CMPA) resonator as a crack sensor. The radar cross section spectrum of the chipless RFID sensor tag generates four resonant frequencies from the dipole resonators and a resonant frequency from the CMPA resonator. Simulation and experimental results show that the resonant frequency shift of the CMPA is a useful feature to indicate the crack orientation and the crack width on a metallic structure. The direction of the resonant frequency shift represents the orientation of the crack, while the magnitude of the resonant frequency shift is proportional to the width of the crack. Furthermore, the experimentation with a natural fatigue crack sample proves that the proposed sensor tag is capable of detecting submillimeter cracks.
\end{abstract}

Index Terms - Chipless radio-frequency identification (RFID), microstrip patch antenna, structural health monitoring (SHM), wireless crack sensor.

\section{INTRODUCTION}

$\mathbf{S}$ TRUCTURAL health monitoring (SHM) is the integration of sensor technologies and the Internet of Things (IoT) to implement automatic detection systems of structural damages on mechanical and civil infrastructures [1], [2]. The acquired information from IoT-based SHM systems can benefit us in life-cycle assessments, asset managements, and preventing failures on invaluable infrastructures such as bridges, wind turbines, aircrafts, and pipelines [3]. Without SHM systems, unmonitored cracks due to fatigue or corrosion on metallic structures may cause hazardous incidents and therefore jeopardize human safety. This is of the uttermost importance to monitor the health condition of safety-critical infrastructures,

Manuscript received June 9, 2017; revised September 22, 2017; accepted November 28, 2017. Date of publication March 7, 2018; date of current version May 4, 2018. This work was supported in part by the Engineering and Physical Sciences Research Council under the Novel Sensing Networks for Intelligent Monitoring Project EP/J012343/1. The work of A. M. J. Marindra was supported by the Indonesia Endowment Fund for Education within the Ministry of Finance, Indonesia. (Corresponding author: Gui Yun Tian.)

The authors are with the School of Engineering, Newcastle University, Newcastle upon Tyne NE1 7RU, U.K. (e-mail: a.m.j.marindra2@ newcastle.ac.uk; g.y.tian@ncl.ac.uk).

Color versions of one or more of the figures in this paper are available online at http://ieeexplore.ieee.org.

Digital Object Identifier 10.1109/TMTT.2017.2786696 in order to prevent catastrophic failures, also to reduce manual maintenance costs. A widely known solution for metal inspection is to utilize nondestructive testing and evaluation (NDT\&E) techniques, e.g., liquid penetrant testing [4], ultrasonic testing [5], eddy current testing [6], thermography testing [7], and microwave waveguide testing [8], which have shown good detection sensitivity and reliability. However, these close-range inspection approaches are time-consuming, cost much in labors, and practically too cumbersome for SHM applications [9].

To bridge the gap between NDT\&E and SHM, radiofrequency identification (RFID)-based sensor systems are attractive because RFID is wireless, passive, and relatively low cost [10], [11]. Caizzone and DiGiampaolo [12], Yi et al. [13], and Zhang et al. [14] have investigated the use of RFID tag antennas as sensors, especially the ultrahigh frequency RFID, for metal crack detection and characterization. It was reported that, by using the phase shift feature from a coupled dipole RFID tags, a submillimeter crack can be detected from 1.5-m distance [12]. A longer reading range, roughly $2.1 \mathrm{~m}$, is achieved with a folded rectangular patch antenna, but the size of the sensor tag is big [13]. For miniaturization purpose, a 3-by-3-cm size 3-D folded dipole RFID tag, reportedly, can detect a crack from 1-m distance using the backscattered power feature in conjunction with principal component analysis (PCA) to reduce the environmental effects [14]. Although the studies have proved the feasibility; however, the use of application specific integrated circuit makes chipped RFID sensor tags not cost-effective and unable to work in harsh environments such as in hightemperature condition. Furthermore, the detection capability of chipped RFID sensor systems is limited to only one parameter detection, i.e., the crack width due to the fact that the chipped RFID standards operate in a narrowband spectrum.

Chipless RFID, the next generation of RFID, offers several advantages over the chipped RFID in terms of cost, simplicity, printability, and ability to work in high-temperature environments [15]-[17]. Therefore, chipless RFID recently has drawn a great attention in sensor applications [18], [19]. One notable progress is the multiparameter frequency signaturebased chipless RFID sensor having a capability to detecting multiple physical variables [20]. The idea of using chipless technologies for crack detection is relatively new. Previously, the time-domain reflectometry-based chipless RFID with delay-line tag structure [21], the frequency-selective surfacebased sensor [22], and the harmonic tag using band-stop filter structure [23] have been observed for detecting cracks on nonmetallic structures. Patch antenna is chipless and has been 


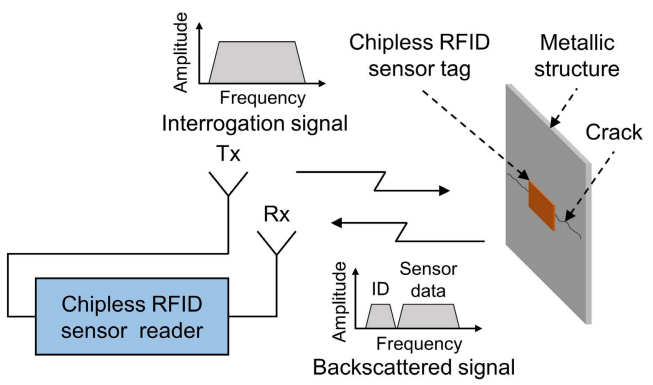

Fig. 1. Principle of chipless RFID sensor system for crack detection and characterization on a metallic structure.

studied for metal crack detection, but unlike the RFID sensor systems, antenna sensors need to be read by wire from the antenna terminal [24]. Nonetheless, as per authors' knowledge, utilization of the frequency signature-based chipless RFID for metal crack detection has not been investigated.

This paper reports a novel use of the frequency signaturebased chipless RFID sensor tag for metal crack detection and characterization. The proposed sensor tag operates in ultra-wideband (UWB) frequency, integrating dipole resonators as the ID encoders and a circular microstrip patch antenna (CMPA) resonator as the crack sensor. By using the resonant frequency shift of the CMPA, the proposed sensor can detect and characterize two crack parameters, i.e., crack orientation and crack width. This multiparameter detection capability has never been achieved by chipped RFID sensors. Crack width is a crucial parameter to signify the severity of a crack, while crack orientation is useful for knowing the crack progression. The direction of resonant frequency shift represents the crack orientation, while its magnitude is proportional to the crack width. With this multiparameter detection capability, we have shown a remarkable potential of chipless RFID sensor system for metal crack detection and characterization.

\section{CRACK Detection Approach}

The working principle of a chipless RFID sensor system for crack detection and characterization is illustrated in Fig. 1. The sensor reader interrogates the sensor tag mounted on a metallic structure by transmitting a broadband signal through the Tx antenna. Reflection from the sensor tag and the metal results in a backscattered signal, which will be received by the Rx antenna. Next, the sensor reader performs signal processing and feature extraction to identify the tag ID as well as to read the sensor information. In this system, the sensor tag is an important part to shape the backscattered signal and to form a unique signature in the frequency domain, which contains the sensing features. The reader will interpret the features so that it can sense if any crack presented on the monitored metallic structure.

Since the concern of this paper is to detect crack on metallic structures, a design requirement to meet is that the sensor tag must be "metal-mountable." A metal-mountable sensor tag design needs to consider the metal as an integral part of the sensor tag. In this case, two-layer chipless RFID tag

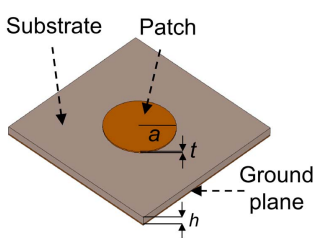

(a)

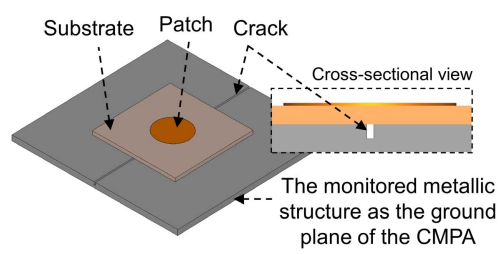

(b)
Fig. 2. CMPA structure (a) Typical configuration. (b) Modified configuration when the antenna applied for crack sensor on a metallic structure.

designs backed with a ground plane can operate well on metal. The reason is that the metallic surface, on which the tag is mounted, acts similarly as the tag's ground plane [25], [26]. The following designs are some of the reported chipless RFID tag structures with a ground plane: dipole [27], folded dipole [28], stepped impedance resonator [29], closed-loop resonator [30], [31], and microstrip patch [32]. Among the feasible metal-mountable designs, this paper takes an interest in CMPA by seeing that a study has revealed its good performance for omnidirectional strain detection [33]. The CMPA sensor has shown a clear difference when the strain is applied with $0^{\circ}$ orientation and $90^{\circ}$ orientation. The resonant frequency will shift toward lower frequencies for $0^{\circ}$-oriented strain and will shift toward higher frequencies for $90^{\circ}$-oriented strain. This evidence is a motivation to assume that a circular shape with its geometric uniformity would also have good results for detecting different crack orientations.

A typical structure of CMPA is shown in Fig. 2(a). Theoretically, the supported modes and the resonant frequency of a CMPA can be found by treating the patch, the ground plane and the material between them as a circular cavity [34]. It is noteworthy that the fundamental resonant frequency of a CMPA will not change significantly with an external wireless excitation [35]. When a CMPA is applied for a metal crack sensor, only a circular patch is printed on the top layer of the substrate while the surface of the monitored metallic structure is considered as the ground plane, as illustrated in Fig. 2(b). Consequently, the electrical properties and the physical condition of the metal underneath of the patch, such as the presence of crack, affect the characteristics of the CMPA. A metal crack can be defined as an open line on a metallic surface along which it has split yet without breaking apart the structure. The presence of a crack in the ground plane will modify the formed circular cavity of the CMPA since the crack will emerge an open gap in the ground plane. This change will affect the antenna mode and the antenna effective radius, considering that the substrate thickness and the permittivity need to take into account of the presented air gap due to the crack. Since the resonant frequency is dependent to the antenna effective radius, the presence of crack can be detected by using the resonant frequency shift of the CMPA.

\section{Chipless RFID SEnsor TAG Design}

Layout and dimension of the proposed chipless RFID sensor tag are presented in Fig. 3 and Table I, respectively. The sensor tag is designed on a Taconic CER-10-0500 laminate with the 


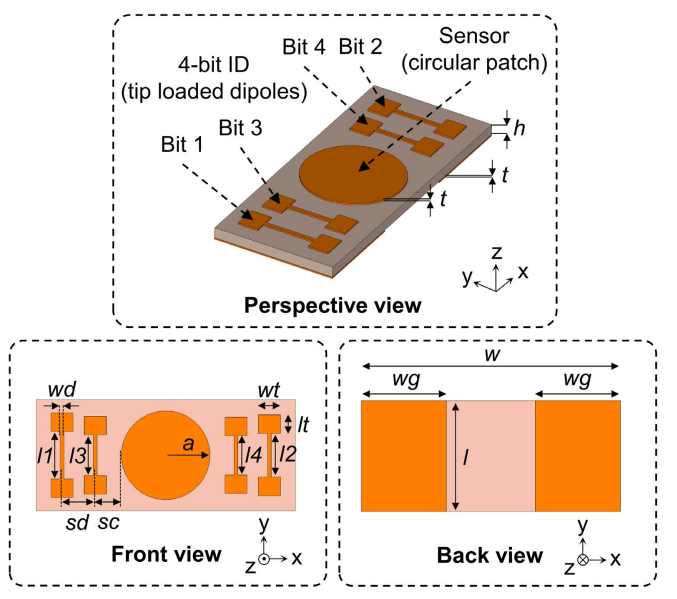

Fig. 3. Layout of the proposed chipless RFID sensor tag.

TABLE I

DiMENSION OF THE PROPOSED CHIPLESS RFID SENSOR TAG (IN mm)

\begin{tabular}{|c|c|c|c|c|c|c|c|c|c|c|c|c|c|c|}
\hline \hline$a$ & $h$ & $l$ & $l 1$ & $l 2$ & $l 3$ & $l 4$ & $l t$ & $s c$ & $s d$ & $t$ & $w$ & $w d$ & $w g$ & $w t$ \\
\hline 6 & 1.27 & 15 & 6.4 & 5.9 & 5.5 & 5.3 & 2.5 & 3.5 & 4.5 & 0.035 & 35 & 0.5 & 11.5 & 3 \\
\hline
\end{tabular}

dielectric constant $\left(\varepsilon_{r}\right)$ of 10 and the loss tangent $(\delta)$ of 0.0035 . A substrate with a high dielectric constant is chosen in order to minimize the size of the sensor tag and maximize the quality factor $(Q)$. The overall size of the chipless RFID sensor tag is $35 \mathrm{~mm} \times 15 \mathrm{~mm}$. The proposed sensor tag consists of two parts of resonators: the sensor part and the ID part. The sensor part of the tag is a CMPA resonator as described in the previous section, while the ID part consists of four tiploaded dipole resonators positioned on the left and right sides of the circular patch. These four ID resonators are to generate a binary ID of "1111." On the sensor tag's backside (see tag's back view in Fig. 3), the ground plane is created only at the backside of ID resonators because the ground plane of the circular patch is the monitored metallic structure. The inherent ground plane on the backside of ID resonators is to protect the ID signature so that the ID of the tag will not be affected by the presence of crack on the metallic structure.

Dipole shape is chosen for ID resonators because of its simple geometry and its ability to resonate on a ground plane. A dipole on a ground plane works as a half wavelength resonator and will have a resonant frequency depending on its length. In our proposed design, the dipole shape is modified with the capacitive tip loading structure, by enlarging both dipole's tips, beneficial to shorten the dipole physical length and to reduce mutual coupling between the resonators. The tip-loaded structure reduces the mutual coupling by making the surface current distributed on the ground plane to be more concentrated, and thus less interfering the adjacent resonators. The real impact of this mutual coupling reduction is that the resonators can be placed closer to each other so that the overall size of the sensor tag decreases significantly over the use of normal dipoles. In addition, two dipole resonators with slightly different lengths should not be placed closely as they produce adjacent resonant frequencies. Therefore, two

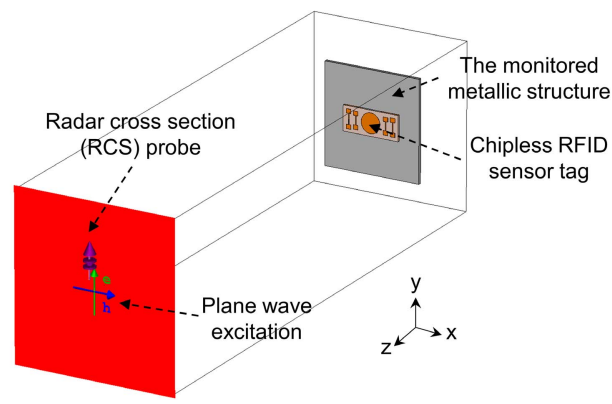

Fig. 4. Simulation setup of the chipless RFID sensor tag.

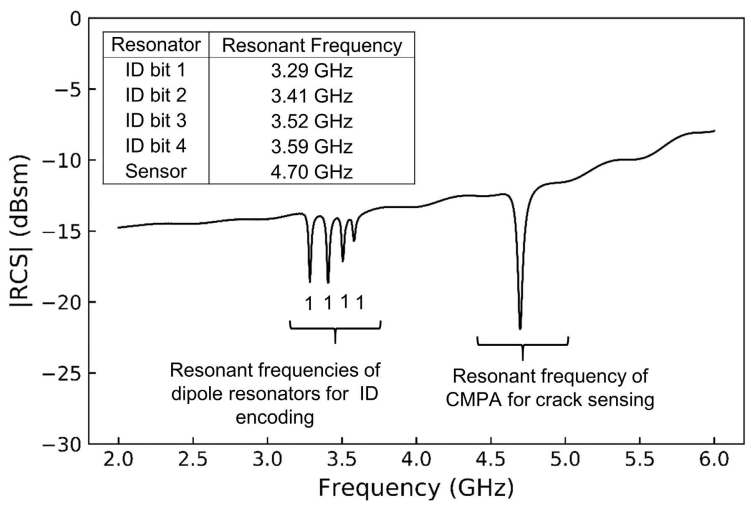

Fig. 5. Simulated RCS spectrum of the chipless RFID sensor tag showing two sets of resonant frequency.

ID resonators are placed on the left side of the circular patch and the other two are placed on the right side. Since multiple resonators are placed on the same surface as an array, there are electromagnetic interactions where the current developed in each resonator also depends on the contributions from the adjacent resonators. The reduction of mutual coupling among the resonators with the mentioned techniques will ensure that each resonator produces resonant frequency distinctly.

The simulation setup in CST Microwave Studio is depicted in Fig. 4. The sensor tag is placed on a metallic plate with a dimension of $60 \mathrm{~mm} \times 60 \mathrm{~mm} \times 2 \mathrm{~mm}$ and an electrical conductivity $(\sigma)$ of $3.56 \times 10^{7} \mathrm{~S} / \mathrm{m}$. Acting as the chipless RFID reader, a plane wave excitation and a radar cross section (RCS) probe are set in the model and positioned at $30 \mathrm{~cm}$ in front of the sensor tag. The plane wave excitation transmits linearly polarized signals to $-z$ direction with its $E$-plane parallel to the $y$-axis and its $H$-plane parallel to the $x$-axis. The setup is simulated within a frequency range from 2 to $6 \mathrm{GHz}$ by using an "open (add space)" boundary setting for all axis directions. The simulated RCS spectrum of the chipless RFID sensor tag is shown in Fig. 5. When a plane wave excites the sensor tag, the RCS shows a frequency selective behavior creating deep notches at particular frequencies. The notches are formed because the conductive structures in the tag resonate the excitation signal at certain frequencies causing some energy at those frequencies to be absorbed before it is reflected. It is obvious that the sensor tag produces two sets of resonant frequency within the frequency range. The first set of resonant 


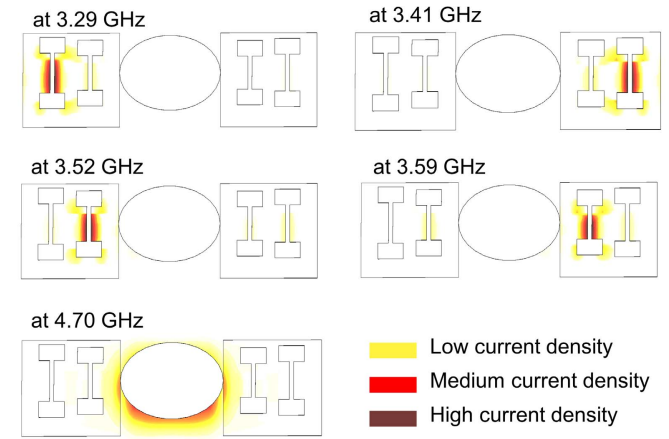

Fig. 6. Surface current distributions of the chipless RFID sensor tag showing the concentration of current at each resonant frequency.

frequency is the ID signature that consists of four resonant frequencies at $3.29,3.41,3.52$, and $3.59 \mathrm{GHz}$. The second set of resonant frequency is the sensor data signature produced by the CMPA at $4.70 \mathrm{GHz}$. The ID signature encodes a tag ID of "1111" since four resonant frequency notches are present. The tag ID can be modified by either eliminating the corresponding ID resonator/s or equalizing its length to a resonator that has ID bit of "1." For example, a tag ID of "1011" can be obtained by eliminating the resonator of bit 2 or equalizing its length to the resonator of bit 1 . Changing the ID bit from " 1 " to " 0 " is essentially done by making the resonant peak absent in the ID data signature.

To relate the resulting resonant frequencies with the tag structure, the surface current distributions of the sensor tag at different frequencies are presented in Fig. 6. It can be seen that each resonator manifests a strong surface current at a particular resonant frequency. At the frequencies of 3.29, 3.41, 3.52, $3.59,4.70 \mathrm{GHz}$, the strongest current appears at the location of dipole resonator of bit 1, bit 2, bit 3, bit 4, and CMPA, respectively. Thus, it is confirmed that the resonant frequencies are contributions from each individual resonator. It is also important to notice that the strong current is more distributed on the ground plane's surface rather than on the resonator's surface. Hence, any change in the RCS spectrum, with respect to physical change on the underlying metallic structure, can be analyzed based on the surface current distribution.

\section{Simulation Study on Metal Crack Detection AND CHARACTERIZATION}

To study how a metal crack affects the RCS of the sensor tag, a crack is created along the monitored metallic structure and the RCS spectrum is simulated for different crack orientations and crack widths. Since the sensor tag is placed at the center of the metallic surface, the constructed crack will pass through the backside of the circular patch. We investigate different widths of metal crack with $0^{\circ}$ orientation (horizontal crack), $90^{\circ}$ orientation (vertical crack), and $45^{\circ}$ orientation (diagonal crack). For each crack orientation, we perform parametric sweep in which the crack depth is fixed at $1 \mathrm{~mm}$, while the crack width is varied from 0 to $3 \mathrm{~mm}$ by $0.1-\mathrm{mm}$ step.

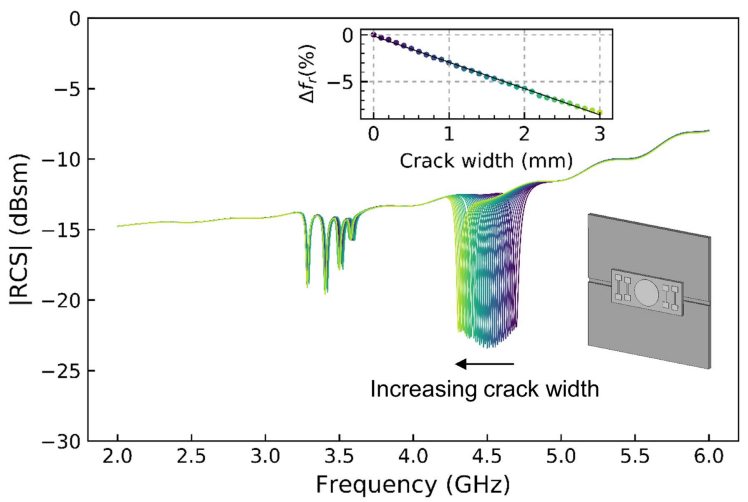

(a)

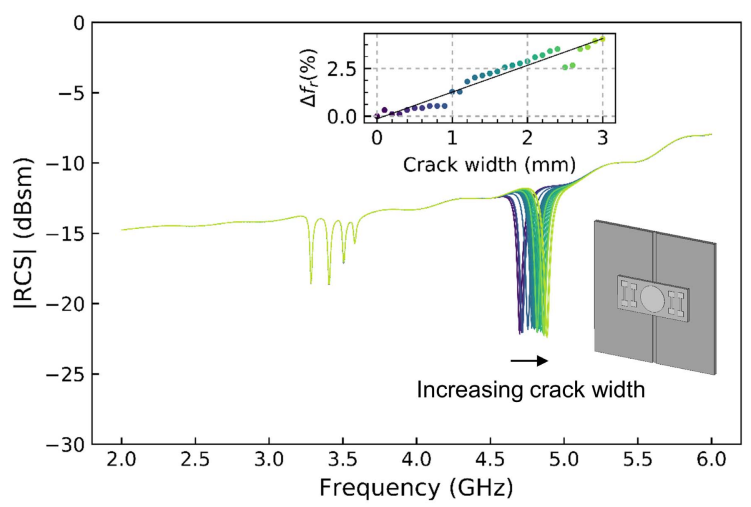

(b)

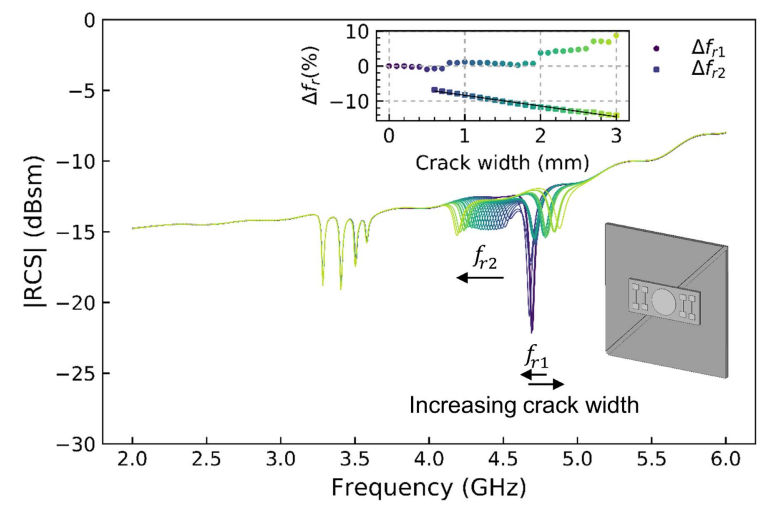

(c)

Fig. 7. Simulated RCS spectra of the chipless RFID sensor tag showing resonant frequency shift for different crack orientations and widths. (a) Horizontal crack. (b) Vertical crack. (c) Diagonal crack.

In metal crack detection, only the sensor's resonant frequency is expected to change according to the orientation and the width of the crack. The simulated RCS spectra of the chipless RFID sensor tag for different crack orientations and widths are presented in Fig. 7. It is apparent that a crack will shift the sensor data signature without affecting the ID signature. In general, a crack will shift the resonant frequency of the CMPA with different directions and magnitudes depending on the orientation and the width of the crack. We use the direction and the magnitude of resonant frequency shift as features to characterize the crack orientation and the crack width, respectively. The normalized magnitude of resonant 
frequency shift $\left(\Delta f_{r}\right)$ in percentage can be represented as

$$
\Delta f_{r}(\%)=\frac{f_{r}^{\text {new }}-f_{r}^{\text {initial }}}{f_{r}^{\text {initial }}} \times 100
$$

where $f_{r}^{\text {initial }}$ is the resonant frequency of the CMPA without the presence of crack and $f_{r}^{\text {new }}$ is the resonant frequency of the CMPA after the crack is presented. The numerical sign of $\Delta f_{r}(\%)$ shows whether the resonant frequency of CMPA shifts toward a lower frequency or a higher frequency.

Fig. 7(a) shows that a horizontal crack will linearly shift the resonant frequency to lower frequencies as the crack width increased. For horizontal crack, the detection sensitivity is approximately $\Delta f_{r}(\%)=-0.28 \%$ or $-13.43 \mathrm{MHz}$ shift per $0.1 \mathrm{~mm}$ increase of the crack width. In opposite to the horizontal crack characterization, a vertical crack will shift the resonant frequency toward higher frequencies as can be seen from Fig. 7(b). In general, the trend of resonant frequency shift for vertical crack detection is linear. The detection sensitivity of vertical crack detection is averagely $\Delta f_{r}(\%)=+0.14 \%$ or $+6.67 \mathrm{MHz}$ shift per $0.1 \mathrm{~mm}$ increase of the crack width. Indeed, the resonant frequency shift is not linear at some crack widths. Nonetheless, we can still justify if a vertical crack exists, as long as the resonant frequency shifts to a higher frequency. The constraint is that, at some degree, the resonant frequency does not represent the width of the vertical crack accurately.

A diagonal crack detection is somewhat more difficult because it relies on two resonant frequencies as shown in Fig. 7(c). Here, the shifts from those two resonant frequencies are named as $\Delta f_{r 1}$ and $\Delta f_{r 2}$. Up to $0.5-\mathrm{mm}$ width, the presence of a diagonal crack can be detected by recognizing the slight shift from $\Delta f_{r 1}$. However, the crack orientation and the crack width cannot be characterized since the trend of $\Delta f_{r 1}$ is irregular. When the crack width is above $0.6 \mathrm{~mm}$, the $\Delta f_{r 2}$ can be identified, and it decreases proportionally as the crack width increased. The $\Delta f_{r 2}$ decreases $-0.31 \%$ or $-15.3 \mathrm{MHz}$ per $0.1 \mathrm{~mm}$ increase of crack width. Hence, a diagonal crack can be detected when two resonant frequencies are present. The $\Delta f_{r 2}$ can be used as a feature to characterize the crack width. A limitation in the diagonal crack detection is that when the crack width is too small, i.e., less than $0.6 \mathrm{~mm}$, the crack may be misinterpreted as if it does not exist or as a horizontal crack.

To understand how the crack shifts the resonant frequency in different ways, we observe the surface current distribution for different crack orientations as presented in Fig. 8. As can be seen from Fig. 8(a), most current paths flowing on a normal metallic structure at the resonant frequency are in the parallel direction with E-plane. Meanwhile, the outer side current paths are curved following the shape of the CMPA, which is circular. When a horizontal crack is present in the ground plane as shown in Fig. 8(b), it will force the current to flow across the crack cavity. The horizontal crack will lengthen the current paths and essentially increases the electrical length of the CMPA. The wider the horizontal crack, the electrical length of the CMPA will be longer. Since the resonant frequency is inversely proportional to the electrical length of an antenna, a horizontal crack will shift the resonant

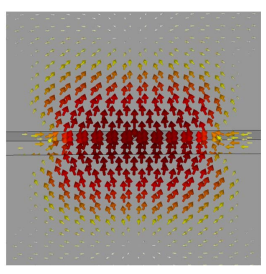

(a)

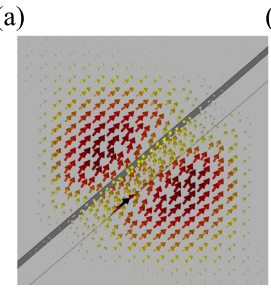

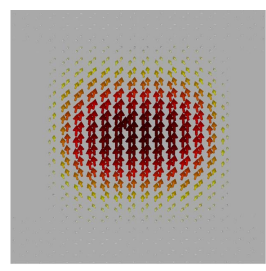

(b)

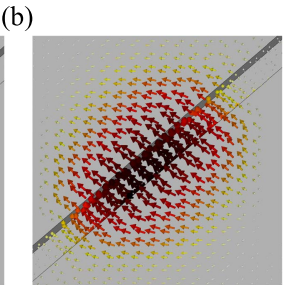

(d)

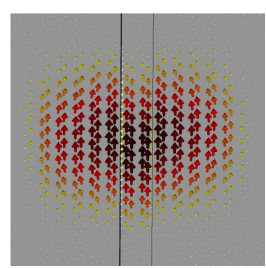

(c)
Fig. 8. Surface current distribution on the metallic structure under the CMPA showing the current flows for different crack orientations. (a) Without crack: at $4.70 \mathrm{GHz}$. (b) With a 2-mm horizontal crack: at $4.43 \mathrm{GHz}$. (c) With a 2-mm vertical crack: at $4.84 \mathrm{GHz}$. (d) With a 2-mm diagonal crack: at $4.30 \mathrm{GHz}$ (left) and $4.77 \mathrm{GHz}$ (right).

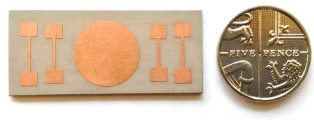

Fig. 9. Fabricated chipless RFID sensor tag.

frequency of CMPA to a lower frequency. In Fig. 8(c), it is shown that a vertical crack will displace some current paths to the bottom surface of the crack. This portion of current, which flows on the crack bottom, will not be taken into account in determining the CMPA effective radius. Thus, due to the current displacement, a vertical crack decreases the electrical length of the CMPA and consequently shifts the resonant frequency to a higher frequency. The diagonal crack combines both phenomena. When a sizeable diagonal crack is present, the CMPA operates in two resonant modes as displayed in Fig. 8(d). The first mode is when the current splits and flows diagonally alongside the crack while there is a small amount of current flowing across the crack. In this mode, the trend of the resulting resonant frequencies from different widths of crack is somehow irregular because the electrical length of the CMPA now depends on not only the length of the diagonal current paths, but also the amount of the current that can flow across the crack. Another mode is created when the current flows diagonally across the crack so that the length of current paths increases as the crack widened. This mode is similar to the phenomenon of the horizontal crack detection in which the resonant frequency will shift to a lower frequency according to the width of the crack.

\section{EXPERIMENTAL VALIDATION AND Discussion}

After investigating the performance of crack detection using simulation, the chipless RFID sensor tag is fabricated and the simulation results are validated through experimental studies. The fabricated chipless RFID sensor tag is depicted in Fig. 9 with a coin for size comparison. We prepare nine artificial crack samples and a natural fatigue crack sample, 


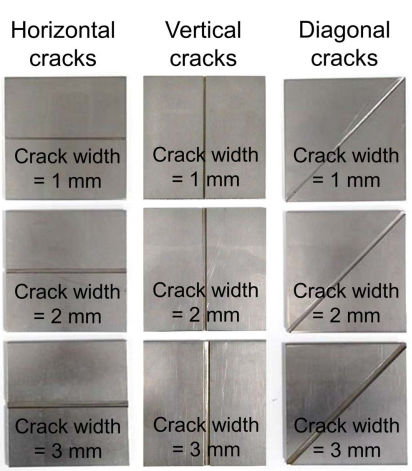

(a)

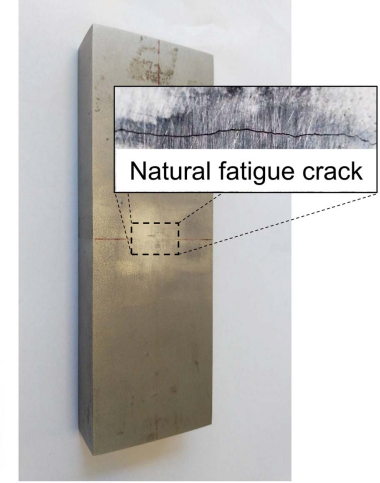

(b)
Fig. 10. Metal crack samples. (a) Artificial crack samples. (b) Natural fatigue crack sample.

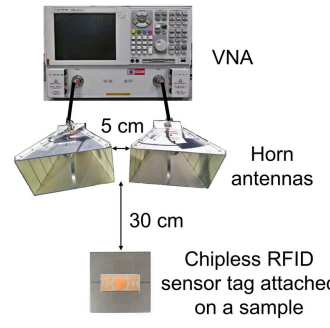

(a)

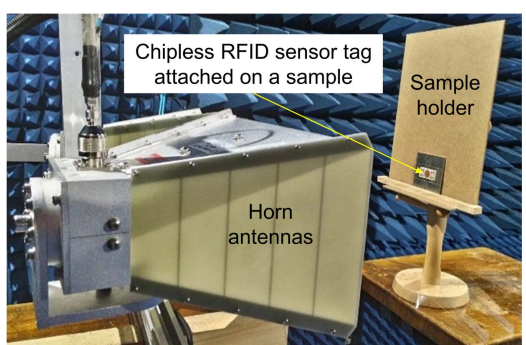

(b)
Fig. 11. Experiment setup using bistatic radar configuration. (a) Diagram of the experimental setup. (b) Photograph showing the positioning of horn antennas and the sensor tag inside an anechoic chamber.

as exhibited in Fig. 10, for validating the results from simulation. The artificial crack samples, shown in Fig. 10(a), are made from aluminum plates with three different crack orientations (horizontal, vertical, and diagonal) and three different crack widths $(1,2$, and $3 \mathrm{~mm}$ ). The natural fatigue crack sample as shown in Fig. 10(b) is a steel bar having dimensions of $170 \mathrm{~mm} \times 50 \mathrm{~mm} \times 20 \mathrm{~mm}$ with a microscopic crack at the center of the surface.

Fig. 11 illustrates the experiment setup using the bistatic radar configuration. As depicted in Fig. 11(a), we use the Agilent 8363B vector network analyzer (VNA) connected to two identical standard gain horn antennas, which are separated by a distance of $5 \mathrm{~cm}$. Although the reader antennas are placed side by side as in the monostatic radar configuration, this tag reading mechanism is considered as bistatic since the phase centers of the horn antennas are comparable to the distance between the horn antennas and the sensor tag. The chipless RFID sensor tag is attached to a metal crack sample by using an adhesive thin transparent tape. The tag and the metal sample are put upright in the sample holder toward the horn antennas and all positioned $30 \mathrm{~cm}$ in front of the horn antennas. The power delivered by the VNA is set as $0 \mathrm{dBm}$ in the entire frequency band with an averaging function activated for 100 measurements to increase the signal to noise ratio.

To obtain RCS from measurement, we need to perform subtractions with the measurement background and to scale the subtracted result using a reference RCS. First, the measured $S_{21}$ of the tag and the sample $\left(S_{21}^{\text {tag+sample }}\right)$ needs to be subtracted with an empty background measurement ( $S_{21}^{\text {background }}$ ). The sample holder should be included in the empty background measurement, as we need to obtain the RCS of tag and sample only. Second, we need to measure $S_{21}$ of a reference object $\left(S_{21}^{\text {ref }}\right)$ having a known RCS from simulation $\left(\mathrm{RCS}_{\mathrm{ref}}\right)$ and to subtract it with the measured empty background. A $50 \mathrm{~mm} \times 50 \mathrm{~mm} \times 2 \mathrm{~mm}$ aluminum plate can be used as the reference object. Hence, the measured RCS can be obtained as [36]

$$
\mathrm{RCS}_{\text {tag }+ \text { sample }}=\left[\frac{S_{21}^{\mathrm{tag}+\text { sample }}-S_{21}^{\text {background }}}{S_{21}^{\text {ref }}-S_{21}^{\text {background }}}\right]^{2} \cdot \mathrm{RCS}_{\text {ref }}
$$

Here, the metal sample has a dominant contribution in forming the RCS. When the size of metal sample is large as compared to the tag, the signal absorption can be too less as compared to the reflection from the sample, thus leads to undetectable resonant frequencies. Hence, the RCS written in (2) has dependence from the size of the metal sample. Fortunately, we can isolate the RCS result from the reflection manifested by the metal sample and leaving the absorption characteristic of the chipless RFID sensor tag. The inversed RCS can be derived by subtracting the measured $S_{21}$ of the tag and the sample ( $\left.S_{21}^{\text {tag+sample }}\right)$ with the measured $S_{21}$ of the metal sample without the tag $\left(S_{21}^{\text {sample }}\right)$, instead of subtraction with $S_{21}^{\text {background }}$. The inversed RCS can be expressed as

$$
\mathrm{RCS}_{\text {tag+sample }}^{\prime}=\left[\frac{S_{21}^{\mathrm{tag}+\text { sample }}-S_{21}^{\text {sample }}}{S_{21}^{\text {ref }}-S_{21}^{\text {background }}}\right]^{2} \cdot \mathrm{RCS}_{\mathrm{ref}} .
$$

The $\mathrm{RCS}_{\text {tag+sample }}^{\prime}$ removes the reflection from metal sample in calculation only, therefore, it still contains the information of the crack because the crack affects the absorptive resonant peak of the CMPA.

\section{A. Readability of the Chipless RFID Sensor Tag}

Chipless RFID sensor tag readability is determined by the observability of the resonant frequencies representing the tag ID and the sensor's resonant frequency. The experimental investigation is started with the measurement of RCS when the chipless RFID sensor tag is attached to a $60 \mathrm{~mm} \times 60 \mathrm{~mm}$ metallic plate without any crack. The reading distance is set at $30 \mathrm{~cm}$ as in the simulation. A comparison between the simulated and the measured RCS spectrum is presented in Fig. 12. As can be seen, the measured RCS agrees very well with the simulated one. Four resonant frequencies representing the tag ID of "1111" and a resonant frequency of CMPA at approximately $4.70 \mathrm{GHz}$ can be observed in the RCS. It means that the chipless RFID sensor tag is definitely readable from $30-\mathrm{cm}$ reading distance. Little discrepancies between the simulated and the measured RCS, especially the resonant frequencies, may be due to the fabrication error.

Next, the readability of the sensor tag is examined with longer reading distances. RCS spectra with reading distances of $30,60,90$, and $120 \mathrm{~cm}$, are measured and the results are shown in Fig. 13. Data of $S_{21}^{\text {tag+sample }}, S_{21}^{\text {background }}$, and $S_{21}^{\text {ref }}$ 


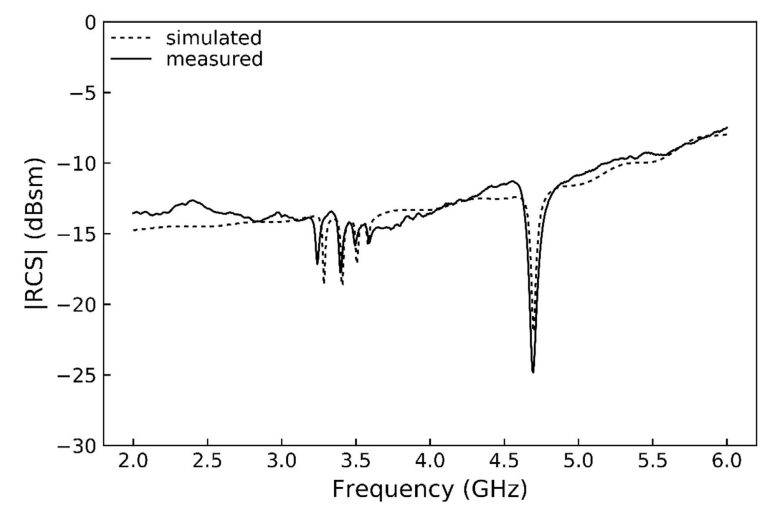

Fig. 12. Comparison between the simulated and the measured RCS spectra of the chipless RFID sensor tag.

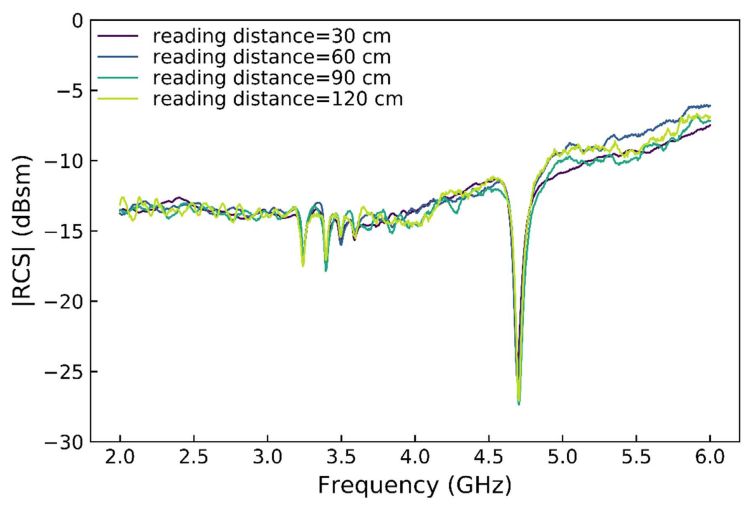

Fig. 13. Measured RCS spectra with different reading distances.

are acquired at every reading distance in the measurements. It is apparent that all resonant frequencies in the RCS are observable from any distance, which means that reading distance does not significantly affect the tag's readability in the anechoic chamber. Indeed, increasing the reading distance will add more ripples into the RCS. Nevertheless, it is not a serious issue as long as the magnitude of the ripples is small as compared to the resonant frequency notches.

In SHM applications, the size of the monitored metallic structures is comparatively much larger than the samples used in this experiment. To examine the readability of the sensor tag on the larger metals, the RCS spectrum is measured at $30-\mathrm{cm}$ reading distance over different sizes of metal. It is obvious from Fig. 14 that the larger metal surface will increase the overall RCS magnitude but will attenuate the resonant frequencies. When the size of metal is $80 \mathrm{~mm} \times 80 \mathrm{~mm}$, the resonant frequencies are still observable. However, they are unobservable if the metal size is $200 \mathrm{~mm} \times 200 \mathrm{~mm}$. The reason is that the considerable amount of reflection from a large metallic surface tends to conceal the absorptive peaks of the resonators. Fortunately, as stated earlier, we can remove the reflection by applying the inversed RCS (RCS') as written in (3). The measured RCS' spectra for different sizes of metal are presented in Fig. 15. It is clear that the resonant frequencies are observable for all metal sizes by using the RCS'. The resulting resonant frequencies in RCS'

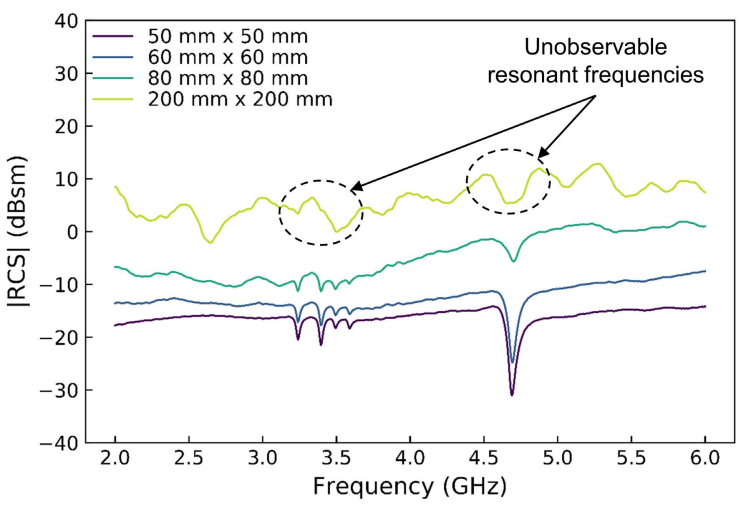

Fig. 14. Measured RCS spectra using (2) of the chipless RFID sensor tag placed on different sizes of metal sample showing unreadable tag if the metal size is $200 \mathrm{~mm} \times 200 \mathrm{~mm}$.

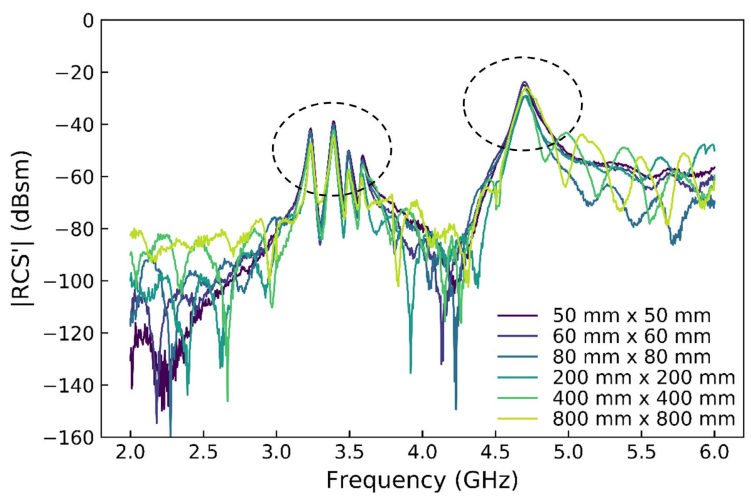

Fig. 15. Measured RCS' spectra using (3) of the chipless RFID sensor tag placed on different sizes of metal sample showing readable tag for any size of metal.

based on (3) are definitely the same as that from RCS based on (2). It is important to note that although the large metal can be calibrated using (3), the measurement becomes more susceptible to noise and sensitive to any movement of the metal in front of the reader antennas. The large metal sample and the other metallic objects inside the chamber, e.g., reader antennas, produces a strong multipath that increases the noise level in the RCS'.

\section{B. Experimental Study With Artificial and Natural Fatigue Cracks}

The simulation results have shown that the direction (positive/negative sign) of resonant frequency shift corresponds to the crack orientation while its magnitude is proportional to the crack width. For validation, RCS measurements are performed with artificial crack samples. We measured the RCS spectrum of three crack widths, i.e., 1, 2, and $3 \mathrm{~mm}$, for each crack orientation. The comparison between the simulated and the measured RCS spectra are given in Fig. 16. It is clear that the simulation and measured results show a very good agreement in trend. In Fig. 16(a), measurement with horizontal crack samples shows that the resonant frequency will shift toward lower frequencies as the crack width increased. The sign of the resonant frequency shift becomes negative and 


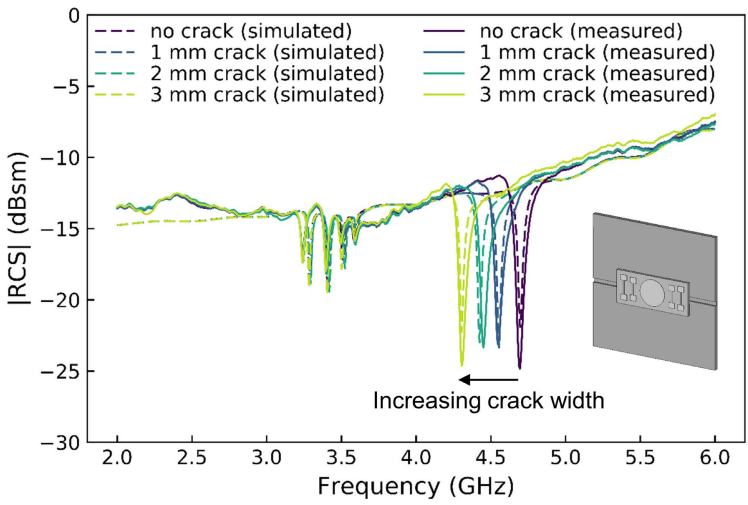

(a)

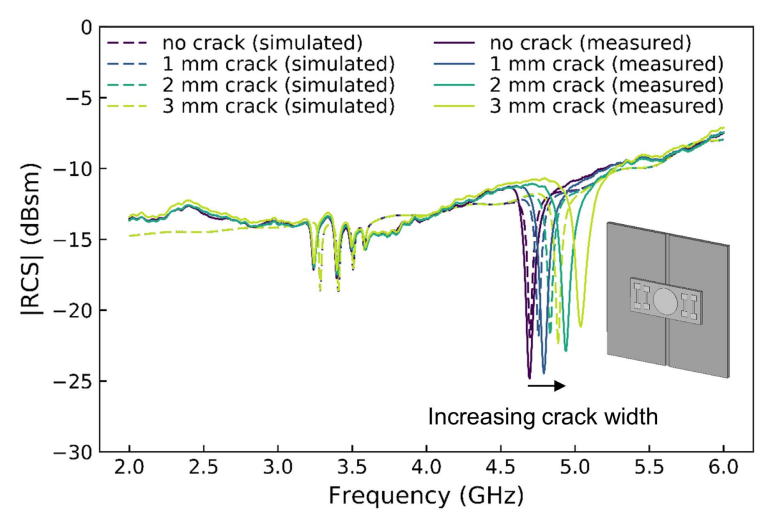

(b)

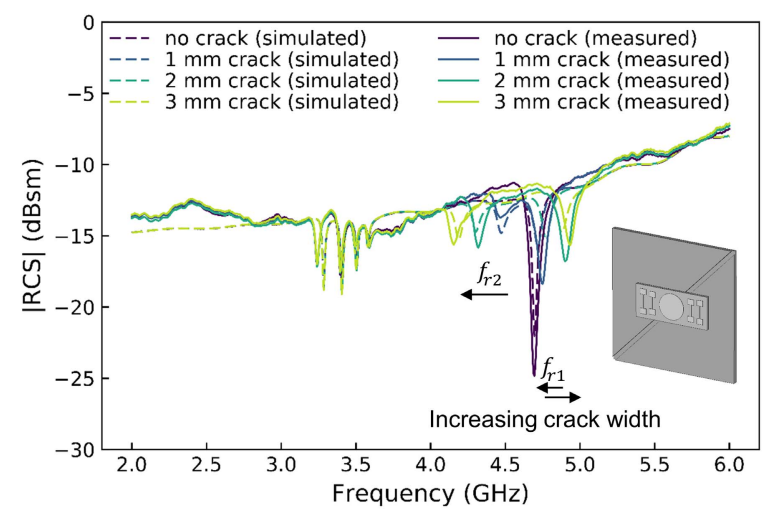

(c)

Fig. 16. Measured RCS spectra of the chipless RFID sensor tag validating the simulation results of resonant frequency shift for different crack orientations and widths. (a) Horizontal crack. (b) Vertical crack. (c) Diagonal crack.

its magnitude represents the width of the horizontal crack. The measured results with vertical crack samples in Fig. 16(b) also provide the same trend with the simulation results. The resonant frequency will shift toward higher frequencies as the crack width increased. The sign of the resonant frequency shift is positive and its magnitude becomes higher when the vertical crack is wider. In addition, the measured results of diagonal crack detection in Fig. 16(c) also demonstrate that the simulation results are valid.

Although the artificial cracks are sufficient for representing real cracks, testing the sensor tag with a natural fatigue crack is necessary to examine the detection reliability. Since the natural

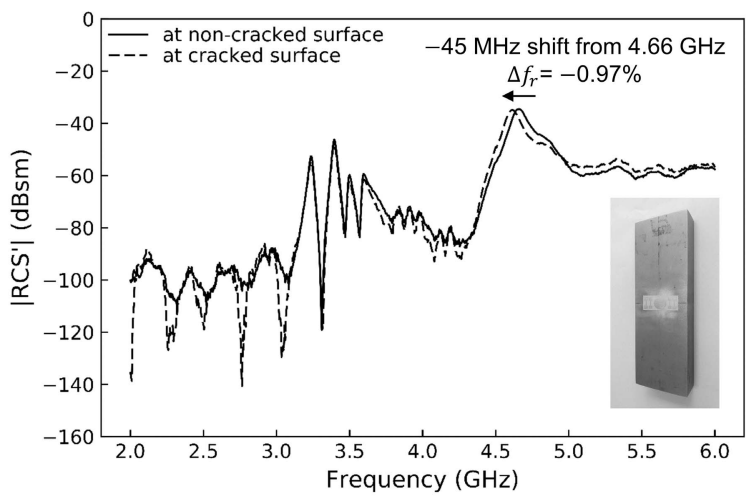

Fig. 17. Measured RCS' spectra showing detection of a microscopic natural fatigue crack by using resonant frequency shift.

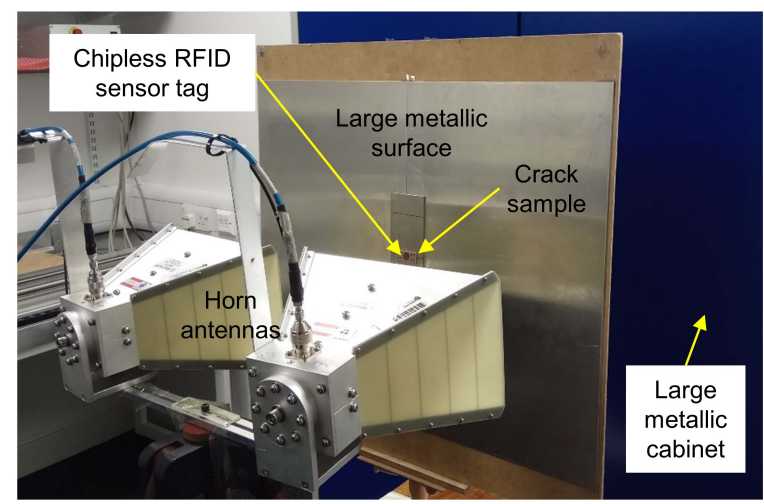

Fig. 18. Experiment setup outside the anechoic chamber.

fatigue crack sample is considerably large in comparison with the sensor tag, we apply the RCS' as in (3) to observe the tag's resonant frequencies. The natural fatigue crack sample is placed upright at the sample holder so that the orientation of natural fatigue crack to detect is horizontal. The RCS' is measured with the tag attached at a noncracked surface and at a cracked surface. The measured RCS' spectra at both locations on the metal surface are shown in Fig. 17. As can be seen, placing the sensor tag at the cracked surface shifts the resonant frequency as much as $-45 \mathrm{MHz}$, or $-0.97 \%$ from the original resonant frequency $(4.66 \mathrm{GHz})$. By assuming that the detection sensitivity is $-0.28 \%$ shift per $0.1-\mathrm{mm}$ crack width obtained from the simulation, the detection result of the natural fatigue crack in the experiment does make sense. The resonant frequency shift of $-0.97 \%$ implies that a horizontal natural fatigue crack with approximately $0.3-\mathrm{mm}$ width is detected.

\section{Experimental Study Outside the Anechoic Chamber}

The experimental validation in the anechoic chamber has shown good results confirming the simulation. Nevertheless, the system implementation for SHM in real environment may not work as good as in the anechoic chamber. To examine the chipless RFID sensor system in real environment, we undertake an experimental study outside the anechoic chamber as depicted in Fig. 18. Three horizontal crack samples having 


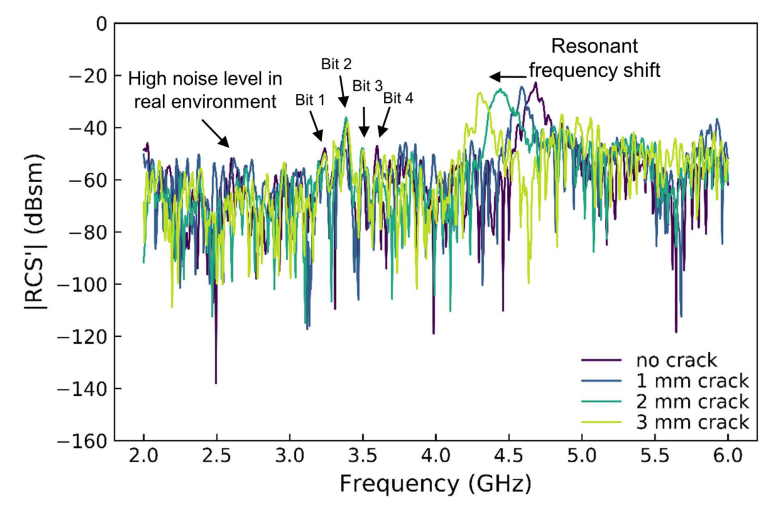

Fig. 19. Measured RCS' spectra showing detection and characterization of horizontal cracks in real environment.

width of 1,2 , and $3 \mathrm{~mm}$ are mounted on an $80 \mathrm{~cm} \times 80 \mathrm{~cm}$ metallic surface, which is positioned $30 \mathrm{~cm}$ away from the horn antennas. The experiment setup is surrounded by various metallic objects such as a large metallic cabinet, computers, VNA, and chairs. In the calculation of RCS' using (3), the reference measurement $S_{21}^{\text {ref }}-S_{21}^{\text {background }}$ with the small aluminum plate is taken from the measurement in the anechoic chamber while the measurement of $S_{21}^{\text {tag+sample }}-S_{21}^{\text {sample }}$ is done in the real environment.

Fig. 19 shows the measurement results outside the anechoic chamber. It is obvious that in such challenging environment, the RCS' suffers from the high noise level, which may obscure the resonant peaks. The large metal area and the surrounding metallic objects produce multipath that increases the level of noise floor in the entire band. Although the resonant peaks from the ID resonators are almost comparable to noise, they are still noticeable at the corresponding frequencies. Likewise, the resonant frequency of the CMPA is easy to observe and the resonant frequency shifts are able to conform with the variation of crack width very well. The result from measurement outside the anechoic chamber suggests that the system is usable in practice but with a limited tag readability. To improve the tag's readability using the current chipless RFID system, the resonator design needs to generate resonant peaks that are able to withstand the high noise level in the real environment.

\section{Limitations and Practical Challenges}

In this paper, the proposed sensor tag can detect the crack only when it is located behind the sensor resonator. It implies that the metal areas behind the ID resonators are blind spots, which should not exist or to be as small as possible. Taking into account the tag ID capacity, which should be high, the ID resonators part should cover less surface area of the monitored metallic structure than the sensor resonator to minimize the blind spots. In addition, the resonant frequencies representing the tag ID should be more visible by making the resonators more absorptive. Obviously, the resonant notches/peaks from dipole resonators in this paper are less visible than that from CMPA. Therefore, the tag ID might be difficult to read in certain conditions. The resonator design for ID part should be improved to have better resonating/absorption characteristics. One more design challenge is related to material and realization of the sensor tag. Chipless RFID sensor tags for metal crack detection have a potential to be fully printed on a flexible and adhesive substrate. The inkjet printing technique with silver/copper nanoparticle ink is commonly used for prototyping. In this case, the impacts of material and electrical properties of conductive ink to the crack detection performance need to be investigated. For industrial applications, the fully printable chipless RFID is a way to enable the crack sensing skin, which can be applied as smart metal coatings in the future.

Despite the sensor tag design, one practical challenge is associated with the robustness of the sensor tag reading. A major drawback of the reading mechanism used in this paper is it needs a calibration measurement with the metallic structure without the attachment of tag as in (3). Nevertheless, performing subtraction with the monitored metallic structure makes the system very sensitive to the positional change and any movement of the structure after the calibration measurement is done. Aside from the metal size problem, the calibration measurement requires the sensor system and the environment to be static and isolated. Consequently, the proposed chipless RFID sensor system is only reliable in an anechoic chamber environment. To be applied in the real environment, the chipless RFID sensor system should be calibration free [37] and robust in facing several factors: the reflection from surrounding objects, the multipath, and the other disturbances that make the system not static. One potential solution is to use depolarizing sensor tags and the cross-polarization reading [38].

Regarding the crack detection performance, the proposed sensor tag has demonstrated its capability in characterizing different crack widths for horizontal, vertical, and diagonal orientation. Enhancements of sensor's capability in classifying the crack size, orientation, and location can be studied further. The challenge is to propose better characterization or quantitative nondestructive evaluation methods applicable for SHM with feature extraction and selection as well as multiple feature fusion based on analysis of the signal magnitude and the phase characteristics. Then, another concern related to the detection performance is to consider the effects of material properties of the monitored structure to the behavior of the sensor tags. Civil metallic structures normally have rough surface and painting layer, which may affect the detection sensitivity and reliability. Therefore, the chipless RFID reader should include mathematical processing such as the PCA [39] in order to separate and analyze multiple interferences from material properties and environment.

Finally yet importantly, RFID array is a further practical challenge aiming at expanding the measurement space to localize metal cracks. In the implementation, a number of chipless RFID sensor tags having different IDs are deployed as an array for large area monitoring. The interference among sensors should be mitigated. Therefore, the chipless RFID sensor reader needs to be embedded with an anticollision algorithm [40] for reading multiple tags and separating sensor data according to the tag ID. Study on chipless RFID array is vital to discover how dense the sensor tags can be placed and read over the monitored metallic structure. 


\section{CONCLUSION}

A novel use of the frequency signature-based chipless RFID for metal crack detection and characterization has been demonstrated and validated by both simulation and experimental studies. We have provided the proof of concept, for the first time, by proposing a chipless RFID sensor tag design integrating dipole resonators as the ID encoders and a CMPA resonator as the crack sensor. The sensor tag generates a frequency signature in the form of RCS showing multiple resonant frequencies, which represent the tag's ID and the sensor data. The resonant frequency shift of the CMPA is found as a feature able to indicate two crack parameters, i.e., crack orientation and crack width, on a metallic structure. The direction of resonant frequency shift can indicate the crack orientation while the magnitude of the shift is proportional to the increase of the crack width. A horizontal crack shifts the resonant frequency of CMPA toward a lower frequency, and oppositely, a vertical crack shifts the resonant frequency of CMPA toward a higher frequency. Meanwhile, a diagonal crack splits the resonant frequency into two, where the shift of one of them has a linear relationship with the crack width. Furthermore, the proposed chipless RFID sensor tag has a high sensitivity. Several megahertz to tens megahertz resonant frequency shift is observable per $0.1 \mathrm{~mm}$ increase of the crack width. Therefore, the proposed sensor tag has a capability to detect a submillimeter natural fatigue crack on a steel bar as expected.

We have highlighted some limitations and practical challenges for further improvements of this application. The major problem with the proposed sensor system is associated with the reading mechanism and the robustness in the real environment. The future work is to redesign the chipless RFID sensor tag having a larger ID capacity and suitable for cross-polarization reading, as well as to implement the sensor reader using a small radar transceiver kit with small directive antennas and signal processing capability. The system should be calibration free, robust in the real environment, and showing more applicability for large infrastructure monitoring. Aside from the sensor tag and system designs, feature extraction, and selection methods as well as signal processing techniques will play important roles to cope with multiple interferences. These issues are already being tackled by the authors.

\section{REFERENCES}

[1] J. P. Lynch, C. R. Farrar, and J. E. Michaels, "Structural health monitoring: Technological advances to practical implementations," Proc. IEEE, vol. 104, no. 8, pp. 1508-1512, Aug. 2016.

[2] A. Zanella, N. Bui, A. Castellani, L. Vangelista, and M. Zorzi, "Internet of Things for smart cities," IEEE Internet Things J., vol. 1, no. 1, pp. 22-32, Feb. 2014.

[3] S. Li, L. Xu, and S. Zhao, "The Internet of Things: A survey," Inf. Syst. Frontiers, vol. 17, no. 2, pp. 243-259, 2015.

[4] F. A. Iddings, "The basics of liquid penetrant testing," Mater. Eval., vol. 44, no. 12 , p. 1364 , Nov. 1986.

[5] B. W. Drinkwater and P. D. Wilcox, "Ultrasonic arrays for nondestructive evaluation: A review," NDT\&E Int., vol. 39, no. 7, pp. 525-541, Oct. 2006.

[6] J. García-Martín, J. Gómez-Gil, and E. Vázquez-Sánchez, "Non-destructive techniques based on eddy current testing," Sensors, vol. 11, no. 3, pp. 2525-2565, Feb. 2011.
[7] V. P. Vavilov and D. D. Burleigh, "Review of pulsed thermal NDT: Physical principles, theory and data processing," NDT\&E Int., vol. 73, pp. 28-52, Jul. 2015.

[8] M. U. Memon and S. Lim, "Review of electromagnetic-based crack sensors for metallic materials (recent research and future perspectives),' Metals, vol. 6, no. 8, p. 172, Aug. 2016.

[9] Y. Yao, S.-T. E. Tung, and B. Glisic, "Crack detection and characterization techniques-An overview," Struct. Control Health Monitor., vol. 21, no. 12, pp. 1387-1413, Dec. 2014.

[10] J. Zhang, G. Y. Tian, A. M. J. Marindra, A. I. Sunny, and A. B. Zhao, "A review of passive RFID tag antenna-based sensors and systems for structural health monitoring applications," Sensors, vol. 17, no. 2, p. 265, Jan. 2017

[11] Z. Meng and Z. Li, "RFID tag as a sensor-A review on the innovative designs and applications," Meas. Sci. Rev., vol. 16, no. 6, pp. 305-315, Dec. 2016.

[12] S. Caizzone and E. DiGiampaolo, "Wireless passive RFID crack width sensor for structural health monitoring," IEEE Sensors J., vol. 15, no. 12, pp. 6767-6774, Dec. 2015.

[13] X. Yi, C. Cho, J. Cooper, Y. Wang, M. M. Tentzeris, and R. T. Leon, "Passive wireless antenna sensor for strain and crack sensingElectromagnetic modeling, simulation, and testing," Smart Mater. Struct., vol. 22, no. 8, p. 085009, Aug. 2013.

[14] J. Zhang, G. Y. Tian, and A. B. Zhao, "Passive RFID sensor systems for crack detection \& characterization," NDT\&E Int., vol. 86, pp. 89-99, Mar. 2017.

[15] S. Preradovic and N. C. Karmakar, "Chipless RFID: Bar code of the future," IEEE Microw. Mag., vol. 11, no. 7, pp. 87-97, Dec. 2010.

[16] S. Tedjini, N. Karmakar, E. Perret, A. Vena, R. Koswatta, and R. E-Azim, "Hold the chips: Chipless technology, an alternative technique for RFID," IEEE Microw. Mag., vol. 14, no. 5, pp. 56-65, Jul./Aug. 2013.

[17] N. C. Karmaker, "Tag, you're it radar cross section of chipless RFID tags," IEEE Microw. Mag., vol. 17, no. 7, pp. 64-74, Jul. 2016.

[18] S. Preradovic and N. Karmakar, "Chipless RFID tag with integrated sensor," in Proc. IEEE SENSORS, Nov. 2010, pp. 1277-1281.

[19] A. Vena, L. Sydänheimo, M. M. Tentzeris, and L. Ukkonen, "A fully inkjet-printed wireless and chipless sensor for $\mathrm{CO}_{2}$ and temperature detection," IEEE Sensors J., vol. 15, no. 1, pp. 89-99, Jan. 2015.

[20] E. M. Amin, N. C. Karmakar, and B. W. Jensen, "Fully printable chipless RFID multi-parameter sensor,' Sens. Actuators A, Phys., vol. 248, pp. 223-232, Sep. 2016.

[21] S. Dey, P. Kalansuriya, and N. C. Karmakar, "Chipless RFID based high resolution crack sensing through SWB technology," in Proc. IEEE Int. Microw. RF Conf., Dec. 2014, pp. 330-333.

[22] S.-D. Jang, B.-W. Kang, and J. Kim, "Frequency selective surface based passive wireless sensor for structural health monitoring," Smart Mater. Struct., vol. 22, no. 2, p. 025002, Dec. 2013.

[23] V. Palazzi et al., "Demonstration of a chipless harmonic tag working as crack sensor for electronic sealing applications," Wireless Power Transf., vol. 2, no. 2, pp. 78-85, Sep. 2015.

[24] I. Mohammad and H. Y. Huang, "An antenna sensor for crack detection and monitoring," Adv. Struct. Eng., vol. 14, no. 1, pp. 47-53, Feb. 2011

[25] F. Costa, S. Genovesi, and A. Monorchio, "A chipless RFID readable on metallic objects," in Proc. IEEE Antennas Propag. Soc. Int. Symp., Jul. 2013, pp. 1498-1499.

[26] F. Costa, S. Genovesi, and A. Monorchio, "Chipless RFIDs for metallic objects by using cross polarization encoding," IEEE Trans. Antennas Propag., vol. 62, no. 8, pp. 4402-4407, Aug. 2014.

[27] I. Jalaly and I. D. Robertson, "RF barcodes using multiple frequency bands," in IEEE MTT-S Int. Microw. Symp. Dig., Jun. 2005, pp. 139-142.

[28] J. Machac, M. Polivka, M. Svanda, and J. Havlicek, "Reducing mutual coupling in chipless RFID tags composed of U-folded dipole scatterers," Microw. Opt. Technol. Lett., vol. 58, no. 11, pp. 2723-2725, Nov. 2016.

[29] C. M. Nijas et al., "Low-cost multiple-bit encoded chipless RFID tag using stepped impedance resonator," IEEE Trans. Antennas Propag., vol. 62, no. 9, pp. 4762-4770, Sep. 2014.

[30] F. Costa, S. Genovesi, and A. Monorchio, "A chipless RFID based on multiresonant high-impedance surfaces," IEEE Trans. Microw. Theory Techn., vol. 61, no. 1, pp. 146-153, Jan. 2013 
[31] M. M. Khan, F. A. Tahir, M. F. Farooqui, A. Shamim, and H. M. Cheema, "3.56-bits $/ \mathrm{cm}^{2}$ compact inkjet printed and application specific chipless RFID tag," IEEE Antennas Wireless Propag. Lett., vol. 15, pp. 1109-1112, 2016.

[32] P. Kalansuriya, N. C. Karmakar, and E. Viterbo, "On the detection of frequency-spectra-based chipless RFID using UWB impulsed interrogation," IEEE Trans. Microw. Theory Techn., vol. 60, no. 12, pp. 4187-4197, Dec. 2012.

[33] A. Daliri, A. Galehdar, W. S. T. Rowe, K. Ghorbani, and S. John, "Utilising microstrip patch antenna strain sensors for structural health monitoring," J. Intell. Mater. Syst. Struct., vol. 23, no. 2, pp. 169-182, Jan. 2012.

[34] C. A. Balanis, Antenna Theory: Analysis and Design. Hoboken, NJ, USA: Wiley, 2015.

[35] X. Xu and H. Huang, "Multiplexing passive wireless antenna sensors for multi-site crack detection and monitoring," Smart Mater. Struct., vol. 21, no. 1, Jan. 2012, Art. no. 015004.

[36] A. Vena, E. Perret, and S. Tedjini, "Design of compact and autocompensated single-layer chipless RFID tag," IEEE Trans. Microw. Theory Techn., vol. 60, no. 9, pp. 2913-2924, Sep. 2012.

[37] F. Costa, S. Genovesi, and A. Monorchio, "Normalization-free chipless RFIDs by using dual-polarized interrogation," IEEE Trans. Microw. Theory Techn., vol. 64, no. 1, pp. 310-318, Jan. 2016.

[38] A. Vena, E. Perret, and S. Tedjni, "A depolarizing chipless RFID tag for robust detection and its FCC compliant UWB reading system," IEEE Trans. Microw. Theory Techn., vol. 61, no. 8, pp. 2982-2994, Aug. 2013.

[39] J. Zhang and G. Y. Tian, "UHF RFID tag antenna-based sensing for corrosion detection \& characterization using principal component analysis," IEEE Trans. Antennas Propag., vol. 64, no. 10, pp. 4405-4414, Oct. 2016.

[40] R. Rezaiesarlak and M. Manteghi, "A space-time-frequency anticollision algorithm for identifying chipless RFID tags," IEEE Trans. Antennas Propag., vol. 62, no. 3, pp. 1425-1432, Mar. 2014.

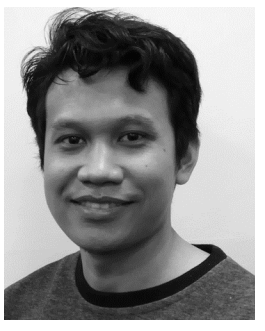

Adi Mahmud Jaya Marindra (S'18) received the degree in electrical engineering from Gadjah Mada University, Yogyakarta, Indonesia, in 2010, and the master's degree in computer engineering from the King Mongkut's Institute of Technology Ladkrabang, Bangkok, Thailand, in 2013. He is currently pursuing the Ph.D. degree in electrical and electronic engineering at the School of Engineering, Newcastle University, U.K.

In 2013, he joined the RF Electronics Research Group, National Electronics and Computer Technology Center, Thailand, where he was involved in printed RFID. Since 2015, he has been a Lecturer with the Kalimantan Institute of Technology, Balikpapan, Indonesia. His current research interests include antennas and propagation, RFID, and wireless sensors.

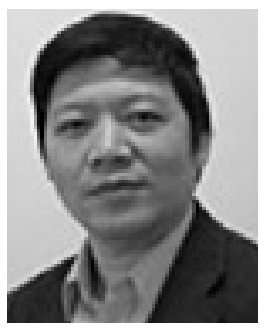

Gui Yun Tian (M'01-SM'03) received the B.Sc. degree in metrology and instrumentation and M.Sc. degree in precision engineering from the University of Sichuan, Chengdu, China, in 1985 and 1988 respectively, and the Ph.D. degree from the University of Derby, Derby, U.K., in 1998.

He was a Lecturer, a Senior Lecturer, a Reader, a Professor, and the Head of the Group of Systems Engineering, University of Huddersfield, Huddersfield, U.K., from 2000 to 2006. Since 2007, he has been with Newcastle University, Newcastle upon Tyne, U.K., where he is a Chair Professor of sensor technologies. He is currently an Adjunct Professor with the School of Automation Engineering, University of Electronic Science and Technology of China, Chengdu, China. $\mathrm{He}$ has coordinated several research projects with the Engineering and Physical Sciences Research Council, the Royal Academy of Engineering, and FP7. He has also collaborated with leading industrial companies such as Airbus,Toulouse, France, Rolls Royce, Derby, BP, London, U.K., nPower, Swindon, U.K., and TWI. 